\title{
Lungenfunktionsmessung bei Kindern vor und nach einem altersadaptierten SCUBA Tauchgang im Schwimmbad
}

\author{
Lung Function Testing in Children before and after an Age-Adapted SCUBA Dive \\ in a Swimming Pool
}

Autoren

Institute
P. Wollin', M. Christmann' ${ }^{1}$, A. Kroker ${ }^{2}$, S. Zielen ${ }^{1}$

1 Allergologie, Pneumologie und Mukoviszidose, Zentrum für Kinder- und Jugendmedizin,

J. W. Goethe-Universität Frankfurt am Main

2 Pneumologische Schwerpunktpraxis Kroker, Koblenz eingereicht 29.11.2010 akzeptiert nach Revision 20.12. 2010

\section{Bibliografie}

DOI http://dx.doi.org/ 10.1055/s-0030-1256152

Online-Publikation: 3. 2. 2011

Pneumologie 2011; 65: 308-313 @ Georg Thieme Verlag KG Stuttgart · New York ISSN 0934-8387

\section{Korrespondenzadresse} Dr. med. Martin Christmann Klinikum der Johann Wolfgang Goethe-Universität Zentrum für Kinder- und Jugendmedizin

Allergologie, Pneumologie und Mukoviszidose

Theodor-Stern-Kai 7

60590 Frankfurt am Main

martin.christmann@kgu.de

\section{Zusammenfassung \\ $\nabla$}

Einleitung: Immer mehr Kinder sind im Tauchsport aktiv. Atemwegsverengungen beim Tauchen mit der Druckluftflasche sind sehr gefährlich und können unter Wasser ein pulmonales Barotrauma mit arterieller Gasembolie hervorrufen. Statistiken zeigen, dass Kinder davon verstärkt betroffen sind. Da es über die Lungenfunktion bei Kindern während eines Tauchgangs sehr wenige Daten gibt, ist es das Ziel der vorliegenden Studie, diesbezüglich neue Erkenntnisse zu gewinnen.

Material und Methoden: Bei 41 Kindern im Alter von 8-14 Jahren wurde vor und nach einem altersadaptierten SCUBA Tauchgang im Schwimmbad eine Lungenfunktionsmessung (Spirometrie und Messung des Residualvolumens) durchgeführt.

Ergebnisse: Die dynamischen exspiratorischen Lungenfunktionsparameter $\mathrm{FEV}_{1}(\mathrm{p}<0,01), \mathrm{FEV}_{1} /$ VC $(p<0,05), \quad$ MEF 75\% $(p<0,05), \quad$ MEF 50\% $(\mathrm{p}<0,01)$ und MEF 25\% $(\mathrm{p}<0,05)$ verringerten sich signifikant. Es waren keine statistisch signifikanten Veränderungen des Residualvolumens nachweisbar. 5 Probanden (12,2\%) hatten einen $\mathrm{FEV}_{1}$-Abfall $>10 \%(12 \%-21 \%)$.

Schlussfolgerung: Die Mehrheit der Kinder $(87,8 \%)$ wies keine bedeutsamen Lungenfunktionsänderungen auf. 5 Kinder zeigten eine ausgeprägte Reduzierung der FEV ${ }_{1}$. Hinweise sprechen für eine bronchiale Hyperreagibilität(BHR) als entscheidenden Faktor. Kinder mit Asthma bronchiale oder BHR sollten nicht tauchen. Sehr gründliche Tauchtauglichkeitsuntersuchungen (inkl. unspezifischer bronchialer Provokationstestung) sind daher notwendig.

\section{Abstract \\ $\nabla$}

Background: The number of children that SCUBA dive is increasing. Airway narrowing while SCUBA diving can cause dangerous complications like pulmonary barotrauma and arterial gas embolism. Statistics show that children are at an increased risk. Since data are scarce, the goal of this study was to gain new knowledge about acute lung function changes in children while SCUBA diving.

Material and Methods: 41 children aged 8-14 years underwent lung function testing (spirometry and residual volume measurement) before and after a single age-adapted SCUBA dive in a swimming pool.

Results: A significant reduction of the dynamic expiratory lung function parameters $\mathrm{FEV}_{1}$ $(\mathrm{p}<0.01), \mathrm{FEV}_{1} / \mathrm{VC}(\mathrm{p}<0.05), \operatorname{MEF} 75 \%(\mathrm{p}<0.05)$, MEF 50\% $(p<0.01)$ und MEF 25\% $(p<0.05)$ was measured. No statistically significant change of the residual volume was found. A decrease of $\mathrm{FEV}_{1}>10 \%(12 \%-21 \%)$ was found in 5 children (12.2\%).

Conclusion: The majority of the children (87.8\%) did not show any relevant lung function changes. Five children had a considerable reduction of $\mathrm{FEV}_{1}$. Signs indicate the importance of bronchial hyperreactivity (BHR) as a key factor. Children with asthma or BHR should not SCUBA dive. A detailed medical examination is recommended (including an unspecific bronchial provocation test) before starting to dive. 


\section{Einleitung}

Tauchen ist zu einem Trendsport geworden. Die Zahl der Sporttaucher in Deutschland ist inzwischen auf über eine Million angestiegen [1]. Auch eine zunehmende Zahl von Kindern taucht mit der Druckluftflasche (engl.: „SCUBA-diving“). Dieser Trend wird von den großen Tauchsportorganisationen unterstützt, und spezielle Tauchkurse werden schon für Kinder ab dem 8. Lebensjahr angeboten. Beim Tauchen ist der menschliche Körper enormen Belastungen und Risiken ausgesetzt und immer wieder kommt es zu Tauchunfällen mit zum Teil schwerwiegenden Folgen [2]. Die ärztliche Tauchtauglichkeitsuntersuchung ist von großer Bedeutung, um Tauchaspiranten mit gesundheitlichen Einschränkungen frühzeitig zu erkennen und sie kompetent zu ihrer Sicherheit beraten zu können [3]. Ein gesundes Atemsystem ist beim Tauchen besonders wichtig, da Einschränkungen unter Wasser sehr gefährliche Folgen haben können. Die arterielle Gasembolie (AGE) infolge eines pulmonalen Barotraumas ist eine gefürchtete Komplikation des Drucklufttauchens. Hier kommt es zur Überdehnung und zum Einriss des Lungenparenchyms, wenn sich z. B. beim Auftauchen die Atemluft in der Lunge aufgrund des verminderten Umgebungsdrucks ausweitet und nicht abgeatmet werden kann. Gründe hierfür können eine Atemwegsverengung sowie unregelmäßiges oder zu langsames Ein-/Ausatmen sein [4]. Eine Studie, die Tauchunfälle bei Kindern untersuchte, hat festgestellt, dass eine arterielle Gasembolie bei Kindern 2,5-mal häufiger auftritt als bei Erwachsenen [5]. Ob Kinder verstärkt von Atemwegsverengungen unter Wasser betroffen sind oder fehlerhaftes Verhalten beim Auftauchen zu dieser hohen Quote führt, ist noch unklar.

Speziell das Atemwegssystem von Kindern unterscheidet sich morphologisch und physiologisch stark von dem des Erwachsenen. Weltweit gibt es nur 2 Studien, welche die Lungenfunktion von Kindern direkt nach einem Tauchgang gemessen haben. Demnach ergeben sich Hinweise darauf, dass das Tauchen bei Kindern eine bronchiale Obstruktion verursachen kann [6,7]. Es ist daher die Intention dieser Arbeit, weitere Erkenntnisse über mögliche Lungenfunktionsveränderungen von Kindern beim Tauchen zu gewinnen. Die Ergebnisse sollen dabei helfen, gesicherte Richtlinien für die Tauchtauglichkeitsuntersuchungen bei Kindern aufzustellen, um die Sicherheit beim Tauchen für Kinder zu erhöhen. Vor diesem Hintergrund erfolgte die aktuelle Studie zur Messung der Lungenfunktion (Spirometrie und Messung des Residualvolumens) bei Kindern im Alter von 8-14 Jahren vor und nach einem altersadaptierten SCUBA Tauchgang mit der Frage, ob das Tauchen bei Kindern eine obstruktive Ventilationsstörung und eine Lungenüberblähung verursacht.

\section{Material und Methoden \\ $\nabla$}

\section{Probanden}

In der vorliegenden Studie wurden 44 Kinder im Alter von 8-14 untersucht. Drei dieser Kinder konnten nicht in die „per Protokoll“-Auswertung aufgenommen werden; ein Kind musste wegen Ohrenschmerzen (leichtgradiges Barotrauma des Mittelohres) den Tauchgang vorzeitig abbrechen, zwei Kinder wiesen bei der Lungenfunktionsprüfung unvollständige Messwerte auf. Per Protokoll bestand die Studiengruppe somit aus 41 Kindern (28 Jungen und 15 Mädchen) im Alter von 8-14 Jahren, die vor und nach einem Tauchgang mit der Druckluftflasche lungenfunktionell untersucht wurden. Alle Kinder waren Mitglieder eines
Tauchvereins, nahmen erfolgreich an Tauchkursen teil und hatten bereits Erfahrung beim Tauchen mit der Druckluftflasche. Alle Kinder waren Nichtraucher. Die Charakteristika der Teilnehmer können Tab. 1 entnommen werden.

Tab. 1 Charakteristika der Teilnehmer.

\begin{tabular}{ll} 
Parameter & Alter $\mathbf{8 - 1 4}$ Jahre \\
N (Anzahl) & 41 \\
\hline Alter (Jahre) & $11,1 \pm 2,0$ \\
Größe $(\mathrm{cm})$ & $150,4 \pm 12,2$ \\
Gewicht $(\mathrm{kg})$ & $41,4 \pm 12,1$ \\
BMI $\left(\mathrm{kg} / \mathrm{m}^{2}\right)$ & $17,9 \pm 2,5$ \\
Anzahl bisheriger Tauchgänge & $15,1 \pm 13,4$ \\
Angegeben sind Mittelwerte \pm Standardabweichung. \\
BMI: Body Mass Index; m: männlich; w: weiblich.
\end{tabular}

Alle Kinder nahmen freiwillig an der Tauchstudie teil, eine schriftliche Einverständniserklärung von ihren Eltern und eine gültige ärztliche Tauchtauglichkeitsbescheinigung lagen vor. Die Studie war von der Ethikkommission Mainz genehmigt. Die Kinder hatten die Möglichkeit, die Studie zu jedem Zeitpunkt abzubrechen.

\section{Screening}

Einschlusskriterien:

- Erfahrung beim Tauchen mit der Druckluftflasche

- Mitgliedschaft in einem Tauchverein

- Probanden $\geq 8$ und $\leq 14$ Jahre

- schriftliches Einverständnis der Eltern

- ärztliche Tauchtauglichkeitsbescheinigung

- unauffälliger körperlicher Untersuchungsbefund vor dem Tauchgang

Ausschlusskriterien:

- Alter $<8$ und $>14$ Jahre

- diagnostiziertes Asthma bronchiale oder andere behandlungspflichtige Allergie

- akute oder chronische Erkrankungen des Herzen, der Lunge, der Leber oder des Gefäßsystems

- akuter Infekt bzw. Hinweise für akute Erkältungskrankheiten (z.B. Husten, Schnupfen)

- akute oder chronische Otitis

- Schmerzen (z.B. Zephalgien, Ohrenschmerzen)

- Angst vor dem Tauchgang

\section{Ablauf der Studie}

Zu Beginn der Studie wurde von allen Kindern eine tauchspezifische Anamnese erhoben und eine körperliche Untersuchung durchgeführt. Nach Größen- und Gewichtsmessung wurde eine Spirometrie sowie die Messung des Residualvolumens durchgeführt.

Unter Anleitung des Tauchlehrers tauchten die Kinder anschließend mit der vorbereiteten Tauchausrüstung je nach Alter und Taucherfahrung für ca. 20 min in 3-10 m Tiefe (altersadaptiert: 8-9-jährige: $\max .3 \mathrm{~m}$; 10 -11-jährige: $\max .5$ m; 12-14-jährige: max. $10 \mathrm{~m}$; nach Verband deutscher Sporttaucher (VDST) Regelung). Die Wassertemperatur betrug $28^{\circ} \mathrm{C}$. Die Kinder trugen während des Tauchgangs keinen Tauchanzug. Etwa 15 min nach Beendigung des Tauchgangs wurden die Spirometrie, die Messung des Residualvolumens und die körperliche Untersuchung erneut durchgeführt. 


\begin{tabular}{|c|c|c|c|c|}
\hline Parameter & & Vor dem Tauchgang & Nach dem Tauchgang & \\
\hline $\begin{array}{l}\text { VC } \\
\text { Ist/Soll (\%) }\end{array}$ & $M W \pm S D$ & $89,9 \pm 9,1$ & $88,8 \pm 9,5$ & $p=0,19 ;$ n.s. \\
\hline $\begin{array}{l}\text { FEV }_{1} \\
\text { Ist/Soll (\%) }\end{array}$ & $\mathrm{MW} \pm \mathrm{SD}$ & $93,3 \pm 9,4$ & $90,2 \pm 10,7$ & $\mathrm{p}=0,005^{* *}$ \\
\hline FEV $_{1} /$ VC & $\mathrm{MW} \pm \mathrm{SD}$ & $85,7 \pm 5,4$ & $83,9 \pm 6,7$ & $p=0,039^{*}$ \\
\hline $\begin{array}{l}\text { MEF 75\% } \\
\text { Ist/Soll (\%) }\end{array}$ & $\mathrm{MW} \pm \mathrm{SD}$ & $80,5 \pm 18,1$ & $74,9 \pm 16,0$ & $p=0,012^{*}$ \\
\hline $\begin{array}{l}\text { MEF } 50 \% \\
\text { Ist/Soll (\%) }\end{array}$ & $\mathrm{MW} \pm \mathrm{SD}$ & $85,9 \pm 18,6$ & $79,1 \pm 20,7$ & $\mathrm{p}=0,003^{* *}$ \\
\hline $\begin{array}{l}\text { MEF 25\% } \\
\text { Ist/Soll (\%) }\end{array}$ & $\mathrm{MW} \pm \mathrm{SD}$ & $88,4 \pm 26,2$ & $83,2 \pm 27,0$ & $p=0,048^{*}$ \\
\hline $\begin{array}{l}\text { RV } \\
\text { Ist/Soll (\%) }\end{array}$ & $M W \pm S D$ & $129,5 \pm 32,5$ & $120,9 \pm 26,5$ & $p=0,15 ;$ n.s. \\
\hline
\end{tabular}

Tab. 2 Lungenfunktion vo und 15 min nach dem Tauchgang bei $n=41$ Probanden.

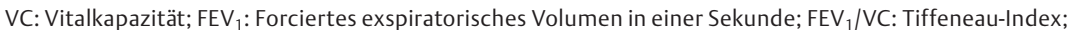

MEF 75\%, 50\%, 25\%: Maximaler exspiratorischer Fluss bei 75\%, 50 \% und 25\% der Vitalkapazität; RV: Residualvolumen;

$\mathrm{MW} \pm$ SD: Mittelwert \pm Standardabweichung; $\mathrm{p}$ : Probabilität; $n$. s.: icht signifikant; ${ }^{*}: \mathrm{p}<0,05 ;{ }^{* *}: \mathrm{p}<0,01$.

\section{Spirometrie}

Für die Bestimmung der mobilisierbaren und dynamischen Lungenvolumina wurde der Spirometer PowerCube ${ }^{\circledR}$ (ohne Body) der Firma GANSHORN MEDIZIN ELECTRONIC GMBH (Niederlauer, Germany) verwendet. An jedem Studientag wurde das System morgens unter Einbezug von Luftdruck, Temperatur und Luftfeuchtigkeit durch den Lungenfunktionstechniker kalibriert. Die Untersuchungen begannen mit einer ausführlichen Instruktion der Probanden. Der Untersuchungsgang wurde dabei beschrieben und demonstriert. Es wurde darauf geachtet, dass die Probanden während der Messungen in aufrechter Position standen. Die Nase war während aller Messungen mit einem Nasenclip verschlossen. Gemessen wurden folgende Parameter: Vitalkapazität (VC), Einsekundenkapazität (forciertes exspiratorisches Volumen in einer Sekunde; $\mathrm{FEV}_{1}$ ), Verhältnis von $\mathrm{FEV}_{1}$ zur VC (TiffeneauIndex; $\mathrm{FEV}_{1} / \mathrm{VC}$ ), maximaler exspiratorischer Fluss bei $75 \%, 50 \%$ und 25\% der forcierten Vitalkapazität (MEF 75\%, 50\%, 25\%). Für alle Probanden wurden die Sollwerte von Zapletal et al. [8] und der European Respiratory Society [9] verwendet.

\section{Residualvolumen}

Zur Messung des Residualvolumens (RV) wurde der Apparat PowerCube ${ }^{\circledR}$-Diffusion der Firma GANSHORN MEDIZIN ELECTRONIC GMBH (Niederlauer, Germany) verwendet. Die Bestimmung des RV wurde mit Hilfe der Helium-Einatemzugs-Einwaschmethode (Single-Breath) durchgeführt. Der Proband atmet hierbei aus einem Gasbeutel einen Atemzug eines Gasgemischs ein (18\% Helium, Rest synthetische Luft). Nach kurzem Luftanhalten wird bei der folgenden langsamen Exspiration der Anteil von Helium in der Ausatemluft analysiert.

\section{Statistische Auswertung}

Die Datenauswertung und graphische Datendarstellung wurde mittels Microsoft ${ }^{\circledR}$ Excel Version 11.0 für Windows ${ }^{\circledR}$ (Microsoft Software Inc., USA), Better Histogram Add-in (Decision Toolworks ${ }^{\mathrm{TM}}$ ) für Microsoft ${ }^{\circledR}$ Excel und GraphPad Prism ${ }^{\circledR}$ Version 4.00 für Windows ${ }^{\circledR}$ (GraphPad Software Inc., San Diego, California/USA) durchgeführt. Die Unterschiede der Lungenfunktionsmesswerte vor und nach den Tauchgängen wurden bei Normalverteilung mittels T-Test (2-tailed) geprüft. Das Signifikanzniveau wurde für alle statistischen Vergleiche auf $\alpha=0,05$ festgelegt.

\section{Ergebnisse}

\section{Lungenfunktionsmessung - Übersicht}

Die Parameter VC, $\mathrm{FEV}_{1}$, MEF 75\%, 50\% und 25\% werden hier aus Vergleichsgründen als prozentuale Werte der individuell erwarteten Sollwerte präsentiert (Ist/Soll). Der Tiffeneau-Index $\mathrm{FEV}_{1} / \mathrm{VC}$ wird als Verhältnis der absoluten Werte angegeben. Die Ergebnisse aller Teilnehmer sind in $\bullet$ Tab. 2 zusammenfassend dargestellt.

\section{Forciertes exspiratorisches Volumen in einer Sekunde $\left(\mathrm{FEV}_{1}\right)$}

Die Einsekundenkapazität (forciertes exspiratorisches Volumen in einer Sekunde; $\mathrm{FEV}_{1}$ ) gibt das Volumen an, das in der 1. Sekunde forcierter Exspiration ausgeatmet werden kann. Der Mittelwert des $\mathrm{FEV}_{1}$ nach dem Tauchgang verringerte sich im Vergleich zur Messung vor dem Tauchgang um 3,1 Prozentpunkte $(\mathrm{p}=0,005) .5$ Kinder zeigten eine Reduktion des $\mathrm{FEV}_{1}$ um mindestens $10 \%(-12 \% ;-13 \% ;-16 \% ;-20 \% ;-21 \%)$. Die genaue Verteilung der individuellen Veränderungen sind $\bullet$ Abb. 1 zu entnehmen.

\section{Residualvolumen}

Das Residualvolumen entspricht dem in der Lunge verbleibenden Luftvolumen am Ende einer forcierten Exspiration. Der Mittelwert des RV nach dem Tauchgang verringerte sich im Vergleich zur Messung vor dem Tauchgang um 8,6 Prozentpunkte $(p=0,15) .63 \%$ der Kinder wiesen nach dem Tauchgang ein niedrigeres RV auf. Bei 13 Probanden verringerte sich das RV um mindestens $20 \%$, bei 19 Probanden betrug die Veränderung weniger als $20 \%$ und bei 9 Probanden vergrößerte sich das RV um mindestens $20 \%$. 3 dieser Probanden verzeichneten eine Zunahme des RV um mehr als 50\%. Bei 6 Kindern (14,6\%) fand sich nach dem Tauchgang ein RV von mindestens 150\%-Ist/Soll.

\section{Korrelationen}

- Abb. 2 zeigt den Zusammenhang (Punktwolke und Regressionsgerade) zwischen den individuellen $\mathrm{FEV}_{1}$-Veränderungen und dem Alter der Probanden. Es konnte hierbei keine statistisch signifikante Korrelation (Pearson Korrelationskoeffizient: $r=0,15 ; p=0,34)$ aufgezeigt werden. 


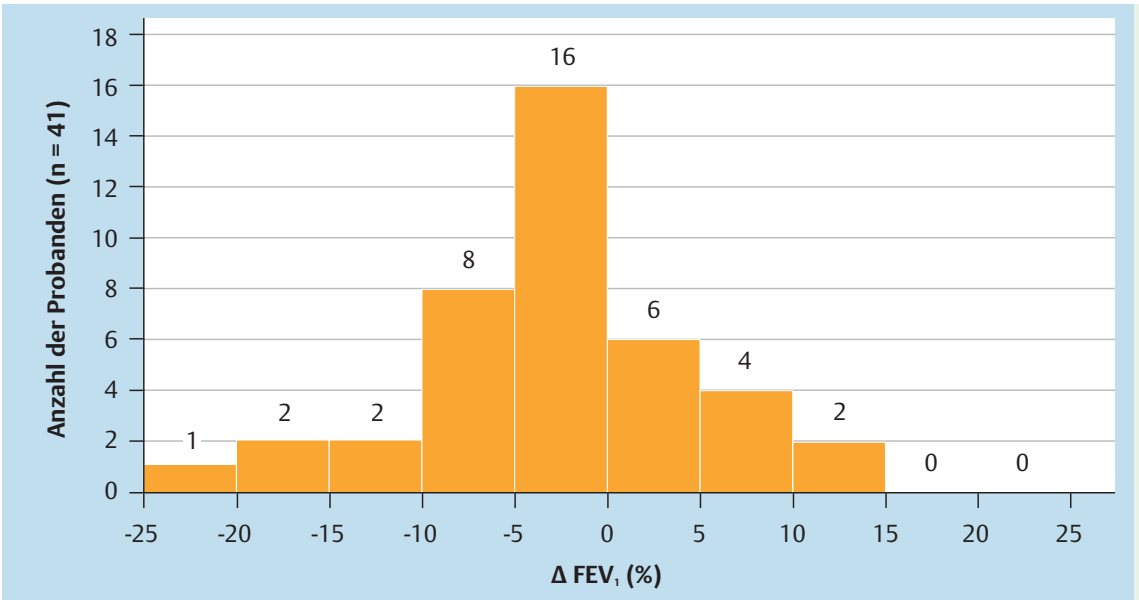

Abb. 1 Individuelle Veränderung der $\mathrm{FEV}_{1}$ in \% nach dem Tauchgang.

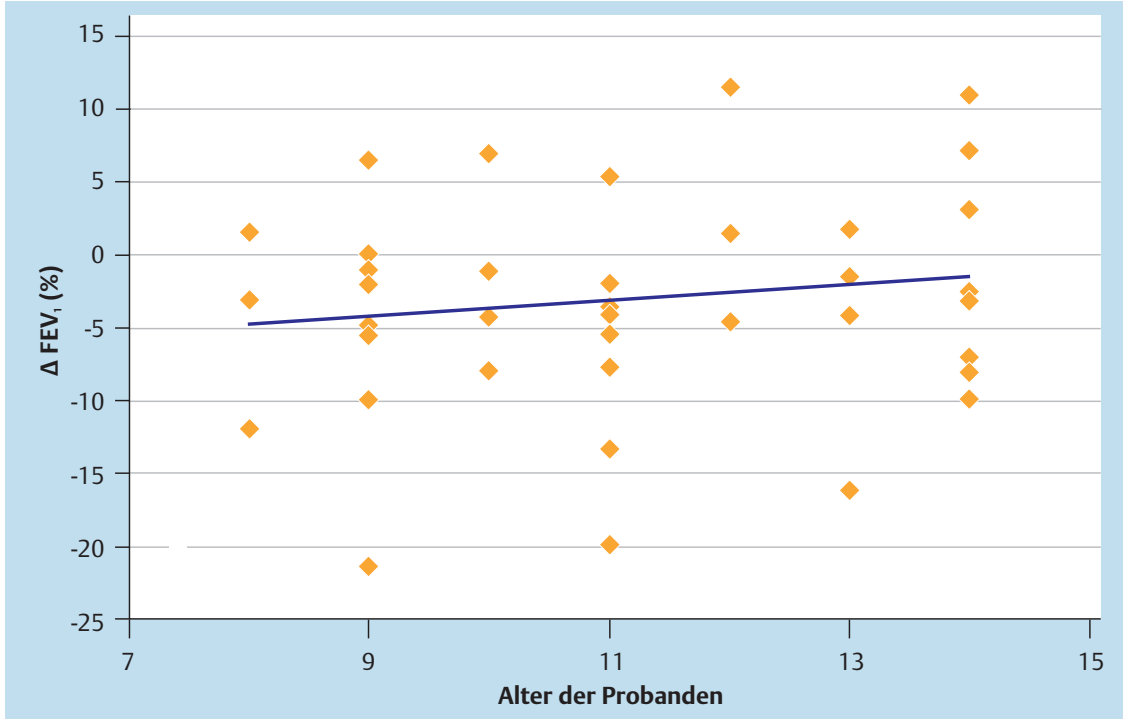

Abb. 2 Zusammenhang zwischen individueller $\mathrm{FEV}_{1}$-Veränderung (\%) und dem Alter der Probanden nach dem Tauchgang.

Es konnte kein statistisch signifikanter Zusammenhang zwischen dem BMI der Kinder und der individuellen $\mathrm{FEV}_{1}$-Veränderung hergestellt werden (Pearson Korrelationskoeffizient: $r=0,04$; $\mathrm{p}=0,8$ ).

Im nächsten Schritt prüften wir die Frage, ob ein Abfall des $\mathrm{FEV}_{1}$ nach dem Tauchgang mit einem Anstieg des RV einhergeht. Es konnte keine statistisch signifikante Korrelation (Pearson Korrelationskoeffizient: $r=0,13 ; p=0,42$ ) aufgezeigt werden.

\section{Diskussion}

\section{$\nabla$}

Eine zunehmende Anzahl von Kindern ab einem Alter von 8 Jahren taucht in Deutschland mit der Druckluftflasche (,SCUBA-diving“). Die Messung der Lungenfunktion bei Tauchern spielt grundsätzlich eine wichtige Rolle, da „Air Trapping“, verursacht durch eine bronchiale Obstruktion, unter Wasser ein pulmonales Barotrauma auslösen kann. Beim Auftauchen dehnt sich das in die Lunge aufgenommene Luftvolumen aufgrund des wieder geringeren Umgebungsdrucks aus. Falls der Taucher zu schnell auftaucht, vergisst regelmäßig auszuatmen oder Hindernisse die Atemwege blockieren, kann es zu einer Überdehnung mit Einrissen des Lungenparenchyms kommen. Letzteres kann z.B. durch die Verengung der Bronchien, durch Reizung und Schwellung der Schleimhaut hervorgerufen werden. Schon eine Druckredu- zierung um 0,1 bar kann bei gleich bleibender Gasmenge zum Einriss des Lungengewebes führen [10].

Aus der Literaturanalyse wird ersichtlich, dass bei gesunden erwachsenen Tauchern in der Regel keine wesentlichen Lungenfunktionsveränderungen direkt nach dem Tauchgang festgestellt wurden [11,12]. Kalte Wassertemperaturen und große Tauchtiefen können jedoch zu Einschränkungen beitragen [13]. Bei Tauchern mit Lungen- und Atemwegserkrankungen wie z. B. Asthma bronchiale, Bronchitis und COPD besteht jedoch eine erhöhte Gefahr, durch „Air Trapping“ ein pulmonales Barotrauma auszulösen $[4,14,15]$. Es ist also wichtig, diese Einschränkungen im Voraus durch gründliche Tauchtauglichkeitsuntersuchungen auszuschließen [3]. Es ist allerdings festzustellen, dass speziell für Kinder nur sehr wenige medizinische und wissenschaftliche Kenntnisse hinsichtlich möglicher pathophysiologischer Einwirkungen des Tauchsports existieren. Weltweit gibt es nur 2 Studien, in denen die Lungenfunktion von Kindern direkt nach einem Tauchgang gemessen wurde. Die Untersuchungen von 18 Kindern im Alter von 9-13 Jahren im Schwimmbad nach einem Tauchgang in $3 \mathrm{~m}$ Tiefe durch Lemaitre et al. [6] und auch die Untersuchung von 16 Kindern im Alter von 10-13 Jahren in einem Badesee nach einem $1 \mathrm{~m}$ und $8 \mathrm{~m}$ tiefen Tauchgang durch Winkler et al. [7] zeigten eine statistisch signifikante Reduktion der $\mathrm{FEV}_{1}$ und der exspiratorischen Flüsse. 
In unserer Studie wurden 41 Kinder körperlich untersucht und vor und nach einem altersadaptierten SCUBA Tauchgang in einem Schwimmbad lungenfunktionell gemessen. Unsere Ergebnisse zeigten keine Veränderung der Vitalkapazität. Die dynamischen exspiratorischen Lungenfunktionsparameter zeigten einen deutlichen Abfall nach dem Tauchgang. Die Parameter $\mathrm{FEV}_{1}$ $(\mathrm{p}<0,01), \quad \mathrm{FEV}_{1} / \mathrm{VC}(\mathrm{p}<0,05), \operatorname{MEF} 75 \%(\mathrm{p}<0,05), \quad$ MEF $50 \%$ $(p<0,01)$ und MEF 25\% $(p<0,05)$ verringerten sich signifikant. Erstmalig wurde in unserer Studie bei Kindern das Residualvolumen (RV) vor und nach einem Tauchgang bestimmt. Es konnte hierbei jedoch keine statistisch signifikante Veränderung des RV festgestellt werden.

Unsere Ergebnisse deuten darauf hin, dass durch das Tauchen bei Kindern eine bronchiale Obstruktion hervorgerufen werden kann.

Bei genauerer Betrachtung der Lungenfunktion jedes einzelnen Kindes wird klar, dass sich die Werte nicht bei allen Kindern in gleichem Maße verschlechtern. Die Mehrzahl der Kinder zeigt keine auffälligen Veränderungen der Lungenfunktion. Bei 5 der 41 Probanden (12,2\%) kam es allerdings zu einem Abfall der $\mathrm{FEV}_{1}$ um mehr als $12 \%$ (12\%-21\%). Drei davon hatten eine Reduktion der $\mathrm{FEV}_{1}$ um mehr als $15 \%$.

Es gab also vereinzelt Kinder, die nach dem Tauchgang deutliche Lungenfunktionsverschlechterungen aufwiesen, während die Mehrzahl der Kinder keine oder nur geringe, tolerierbare Veränderungen zeigten. Für eine mögliche, durch das Tauchen hervorgerufene, bronchiale Obstruktion bei Kindern kommen verschiedene Faktoren in Frage:

Reaktionen bei Kompression und Dekompression des Atemgases aus der Druckluftflasche und der erhöhte Druck unter Wasser führen dazu, dass beim Tauchen trockene, kalte und hyperbare Luft eingeatmet wird [16]. Das Atmen von trockener [17] und kalter [18] Luft unter erhöhtem Druck kann zu einer bronchialen Obstruktion führen. Ein wichtiger Mechanismus hierbei ist der Verlust von Wärme und Wasser über die Atemwege beim Anwärmen und Anfeuchten der Atemluft. Menschen mit erhöhter Atemwegsreagibilität sind davon verstärkt betroffen. Da Kinder im Vergleich zum Erwachsenen eine schnellere Ventilationsrate haben und die Luft unter Wasser eine höhere Dichte aufweist, ventilieren Kinder beim Tauchen eine große Masse kalter und trockener Luft und sind so von den genannten Effekten besonders betroffen. Die Immersion des Körpers in Wasser [19] und das Kühlen der Haut [20] im Wasser können ebenfalls zu Atemwegsverengungen beitragen. Kinder haben entwicklungsphysiologisch bedingt engere Atemwege, und so führen bereits geringe zusätzliche Einengungen der Bronchien bei Kindern schnell zu einer Obstruktion des Lumens. Zudem kann sportliche Anstrengung bei entsprechender Disposition auch alleine zu einer Atemwegsobstruktion führen (excercise induced asthma) [21]. Wieso nun einige Kinder auf diese Faktoren stärker reagieren als andere bleibt eine noch offene Frage. Eine Theorie ist, dass gerade die jüngsten Kinder sich am empfindlichsten zeigen. Es konnte jedoch keine signifikante Korrelation zwischen $\mathrm{FEV}_{1}$-Veränderungen und dem Alter der Kinder aufgezeigt werden ( Abb. 2). Zu berücksichtigen ist, dass die Probanden in den für sie vom VDST empfohlenen Tauchtiefen tauchten (8-9-jährige: max. 3 m; 10 11-jährige: $\max .5 \mathrm{~m}$; 12 -14-jährige: max. 10 m). Genaue Rückschlüsse hinsichtlich der Einwirkungen von Alter und Tauchtiefe sind deshalb nicht leicht. Winkler et al. [7] fanden heraus, dass in ihrer Studie Kinder mit niedrigem BMI einen stärkeren $\mathrm{FEV}_{1}-\mathrm{Ab}-$ fall zeigten. In unserer Studie konnte jedoch keine signifikante
Korrelation zwischen $\mathrm{FEV}_{1}$-Veränderungen und dem BMI der Kinder aufgezeigt werden.

Eine Erklärung für die individuell ungleichen Veränderungen der Lungenfunktionswerte könnte sein, dass bei einigen Kindern eine bronchiale Hyperreagibilität (BHR) vorliegt und sie deshalb empfindlicher auf die beschriebenen Faktoren reagieren. Die BHR kann durch die physikalischen Effekte des Atemgases (hyperbar, kalt, trocken) beim Tauchen verstärkt ausgelöst werden, wodurch eine Atemwegsobstruktion und eine überproportionale Überblähung entstehen kann. In der Übersichtszusammenstellung „Asthma and Recreational SCUBA Diving“ von Koehle et al. [22] wurde festgestellt, dass Tauchen mit Asthma bronchiale zu einer Reduzierung der VC, $\mathrm{FEV}_{1}$ und mittleren exspiratorischen Flüssen führen kann. Des Weiteren sei bei Asthmatikern die „Odds Ratio“ für das Erleiden eines pulmonalen Barotraumas erhöht. Tetzlaff et al. [23] dokumentierten in einer Studie sogar, dass Probanden mit Heuschnupfen möglicherweise beim Tauchen stärker von Lungenfunktionsveränderungen betroffen sind als Gesunde.

Oft erkennen die Betroffenen selber ihre bronchiale Reizempfindlichkeit nicht und unterschätzen geringe Symptome wie intermittierenden Hustenreiz. Im alltäglichen Leben bedarf es bei weitgehender Beschwerdefreiheit trotz vorliegender BHR keiner medizinischen Intervention. Beim Tauchsport jedoch stellt diese eine potenzielle Gefahr dar. Während Kinder mit klinisch nicht ersichtlicher BHR bei üblichen sportlichen Aktivitäten beschwerdefrei sein können, kann es unter Wasser zu Komplikationen kommen. Studien belegen die Gefahren des Tauchens bei Sportlern mit Asthma bronchiale. In einer Fallstudie wurden bei zwei Tauchern mit Asthma zerebrale Luftembolien bereits in den verhältnismäßig geringen Tiefen eines Schwimmbads beschrieben [24]. In einer weiteren Studie wurde bei 3 von 6 Kindern, die beim Tauchen in Hawaii eine arterielle Gasembolie (AGE) erlitten, ein Asthma bronchiale diagnostiziert [5]. Es besteht deshalb grundsätzlich Einigkeit darüber, dass unkontrollierte Asthmatiker aufgrund der erhöhten Unfallgefahr nicht tauchen sollten. Besonders bei Kindern stellt die Diagnose „Asthma bronchiale“ eine explizite Kontraindikation dar. Zwei der Kinder in unserer Studie, die eine $\mathrm{FEV}_{1}$-Reduzierung um mehr als $10 \%$ aufwiesen, wurden zu einem späteren Zeitpunkt erneut auf ihre Tauchtauglichkeit untersucht. Beide unterzogen sich dabei einem unspezifischen Provokationstest mit Methacholin. Hierbei wurde eine deutliche bronchiale Hyperreagibilität nachgewiesen. Diesen Kindern wurde daraufhin vom weiteren Betreiben des Tauchsports abgeraten. Eine BHR der kindlichen Atemwege scheint einen bedeutenden Faktor darzustellen, der Auslöser für Lungenfunktionsverschlechterungen beim Tauchen sein kann und eine reale Gefahr bedeutet. Die BHR bei Kindern kann nicht sicher anamnestisch und durch eine normale Spirometrie festgestellt werden. Aus diesem Grund sollte sie bei Kindern auch durch einen ergänzenden unspezifischen Provokationstest im Rahmen der Tauchtauglichkeitsuntersuchung ausgeschlossen werden.

In der Synopsis der vorliegenden Ergebnisse ist festzustellen, dass die Mehrheit der Kinder (87,8\%) keine bedeutsamen exspiratorischen Flussminderungen aufwiesen. Allerdings fand sich in unseren Untersuchungen bei einem Teil der Kinder (12,2\%) eine bronchiale Obstruktion ( $\mathrm{FEV}_{1}$-Abfall: $\left.\geq 10 \%\right)$ nach dem Tauchgang. Diese Kinder müssen individuell betrachtet werden. Das Vorliegen einer bronchialen Obstruktion erhöht das Risiko, beim Tauchen ein pulmonales Barotrauma zu erleiden. Wahrscheinlich wurden die Atemwegsverengungen der Kinder durch eine unerkannte bronchiale Hyperreagibilität begünstigt. Unsere Ergebnis- 
se stützen die aktuellen Empfehlungen der Gesellschaft für Tauch- und Überdruckmedizin (GTÜM), in welchen empfohlen wird, Kinder mit Asthma bronchiale oder BHR vom Tauchsport auszuschließen. Es ist deshalb anzuraten, Kinder im Rahmen der Tauchtauglichkeitsuntersuchung einem pneumologisch versierten Arzt zur erweiterten Lungenfunktionsuntersuchung mit unspezifischem Provokationstest vorzustellen, da trotz unauffälliger Anamnese und normalen spirometrischen Lungenfunktionsparametern bei Kindern eine BHR vorliegen kann. Hierdurch kann die Sicherheit des Kindertauchens präventiv wesentlich verbessert werden. Weitere Studien sind diesbezüglich auch in Zukunft notwendig, da die Bedeutung der bronchialen Hyperreagibilität und die Möglichkeit einer Langzeitschädigung des kindlichen Bronchialsystems im Tauchsport noch nicht ausreichend erforscht sind.

\section{Interessenkonflikt}

Die Autoren geben an, dass kein Interessenkonflikt besteht.

\section{Literatur}

1 Klingmann C, Tetzlaff $K$, Hrsg. Moderne Tauchmedizin, Handbuch für Tauchlehrer, Taucher und Ärzte. 1. Auflage. Stuttgart: Gentner; 2009

2 Pollock NW, Hrsg. Annual Diving Report - 2008 Edition. Durham, NC: Divers Alert Network; 2008

3 Tetzlaff K, Klingmann C, Muth CM et al., Hrsg. Checkliste Tauchtauglichkeit, Untersuchungsstandards und Empfehlungen der Gesellschaft für Tauch- und Überdruckmedizin (GTÜM) und der Österreichischen Gesellschaft für Tauch- und Hyperbarmedizin (ÖGTH); 1. Auflage. Stuttgart: Gentner; 2009

4 Tetzlaff K, Reuter M. Pneumologische Aspekte der Tauchmedizin. Pneumologie 1998; 52: 489-500

5 Smerz R. Epidemiology and treatment of decompression illness in children and adolescents in Hawaii, 1983 - 2003. SPUMS J 2005; 35: 5 - 10

6 Lemaitre F, Tourny-Chollet C, Hamidouche V et al. Pulmonary function in children after a single scuba dive. Int J Sports Med 2006; 27: 870 874

7 Winkler BE, Tetzlaff K, Muth CM et al. Pulmonary function in children after open water SCUBA dives. Int J Sports Med 2010; 31: 724-730
8 Zapletal A, Samánek M, Paul T. Lung function in children and adolescents, Methods, reference values; 283 tables. Basel: Karger; 1987

9 Quanjer PH, Tammeling GJ, Cotes JE et al. Lung volumes and forced ventilatory flows. Report Working Party Standardization of Lung Function Tests, European Community for Steel and Coal. Official Statement of the European Respiratory Society. Eur Respir J Suppl 1993; 16: 5-40

10 Gesellschaft für Tauch- und Überdruckmedizin e.V. Barotrauma. http://www.gtuem.org/977/Tauchmedizin/Baro-trauma.html. Stand: 15.11.2010

11 Neubauer B, Mutzbauer TS, Struck $N$ et al. Mechanical impedance of the respiratory tract in divers before and after. Eur J Appl Physiol 2005; 95: 454-463

12 Tetzlaff K, Staschen CM, Koch A et al. Respiratory pattern after wet and dry chamber dives to 0.6 MPa ambient pressure. Respir Physiol 1999; 118: $219-226$

13 Tetzlaff K, Friege L, Koch A et al. Effects of ambient cold and depth on lung function in humans after a single scuba. Eur J Appl Physiol 2001; 85: $125-129$

14 Tetzlaff $K$, Reuter M, Leplow B et al. Risk factors for pulmonary barotrauma in divers. Chest 1997; 112: 654-659

15 Russi EW. Diving and the risk of barotrauma. Thorax 1998; 53 (Suppl 2): $520-524$

$16 \mathrm{Ehm}$ OF. Hrsg. Tauchen noch sicherer, Tauchmedizin für Freizeittaucher, Berufstaucher und Ärzte; 9th ed. Cham: Müller Rüschlikon; 2003

17 Thorsen E, Ronnestad I, Segadal K et al. Respiratory effects of warm and dry air at increased ambient pressure. Undersea Biomed Res 1992; 19: $73-83$

18 O'Cain CF, Dowling NB, Slutsky AS et al. Airway effects of respiratory heat loss in normal subjects. J Appl Physiol 1980; 49: 875-880

19 Leddy JJ, Roberts A, Moalem J et al. Effects of water immersion on pulmonary function in asthmatics. Undersea Hyperb Med 2001; 28: 75 82

20 McDonald JS, Nelson J, Lenner KA et al. Effects of the combination of skin cooling and hyperpnea of frigid air in asthmatic and normal subjects. J Appl Physiol 1997; 82: 453-459

$21 \mathrm{McFadden}$ ER Jr., Gilbert IA. Exercise-induced asthma. N Engl J Med 1994; 330: 1362 - 1367

22 Koehle M, Lloyd-Smith R, McKenzie D et al. Asthma and recreational SCUBA diving: a systematic review. Sports Med 2003; 33: 109-116

23 Tetzlaff $K$, Staschen $C M$, Struck $N$ et al. Respiratory effects of a single dive to 50 meters in sport divers with asymptomatic respiratory atopy. Int J Sports Med 2001; 22: 85-89

24 Weiss LD, van Meter KW. Cerebral Air Embolism in Asthmatic Scuba Divers in a Swimming Pool. Chest 1995; 107: 1653-1654 